\title{
The Role of Uric Acid as an Antioxidant in Selected Neurodegenerative Disease Pathogenesis: A Short Review
}

\section{T Settle and H Klandorf*}

West Virginia University, Division of Animal and Nutritional Sciences, Morgantown, West Virginia 26506-6108, USA

*Corresponding author: Hillar Klandorf, West Virginia University, Division of Animal and Nutritional Sciences, Morgantown, West Virginia 26506-6108, USA, Tel: 304293-1897; E-mail: Hillar.Klandorf@mail.wvu.edu

Received date: Apr 21, 2014, Accepted date: May 26, 2014, Published date: May 30, 2014

Copyright: (C) 2014 Settle T, et al. This is an open-access article distributed under the terms of the Creative Commons Attribution License, which permits unrestricted use, distribution, and reproduction in any medium, provided the original author and source are credited.

\begin{abstract}
Uric acid, a potent antioxidant for humans, birds, reptiles, and some primate species, is the end-product of purine degradation that is formed in the xanthine/hypoxanthine reactions catalyzed by xanthine oxidase. Associated with the evolutionary loss of urate oxidase (the enzyme that oxidizes uric acid resulting in the formation of allantoin) and resulting increase in concentrations of uric acid is a prolonged life span. Uric acid is known to scavenge peroxynitrite and other free radicals that can cause an imbalance of oxidants leading to oxidative stress. Uric acid also has a role in protecting DNA from single-strand breaks caused by free radicals in the body leading to a protective effect in neurodegenerative diseases. The brain is particularly vulnerable to oxidative stress as it is considered an 'expensive tissue' with a particularly high metabolic rate and comparatively increased utilization of oxygen. Brain tissue is also high in unsaturated lipids, which makes it more susceptible to free radical damage. Oxidative stress is thus linked to the pathogenesis of neurodegenerative diseases and also ischemic brain injury. In this review, we summarize the function of uric acid in alleviating oxidative damage and providing protection to neural cells during injury and disease.
\end{abstract}

\section{Introduction}

Uric acid has been studied extensively in many physiological and pathological systems including cancer [1] due to its role as a potent plasma antioxidant that scavenges singlet oxygen, peroxy radicals, and hydroxyl radicals. Free radical imbalance within a biological system can result in oxidative damage and inflammation which can, ultimately, increase pathogenesis of disease. The purpose of this review is to link oxidative stress and the ameliorating effects of uric acid with the pathogenesis of disease and present evidence toward the possibility of implementing uric acid as a potential therapeutic agent.

Reactive oxygen species (ROS) are fundamentally free radicals derived from molecular oxygen. Oxygen is required for the generation of all ROS and reactive nitrogen species (RNS) as well as reactive chlorine species [2]. Ground state oxygen, also referred to as the triplet state, is considered to be a bi-radical, meaning that it contains two unpaired electrons in the outer shell. The two electrons exhibit the same spin which enables the oxygen molecule to react with one electron at a time. In a chemical bond, oxygen is not particularly reactive with the two electrons. However, if one of the unpaired electrons becomes excited it can alter its spin state, which results in a singlet oxygen species. The singlet oxygen can react with other pairs of electrons, especially those involved in double bonds, and can become a powerful oxidant [3]

The most commonly known ROS produced in biological systems are the hydroxyl $(\cdot \mathrm{OH})$ radicals, the superoxide radicals $\left(\mathrm{O}_{2}^{-} \cdot\right)$, nitric oxide (NO), peroxynitrite $\left(\mathrm{ONOO}^{-}\right)$, and hydrogen peroxide $\left(\mathrm{H}_{2} \mathrm{O}_{2}\right)$. Free radicals and other ROS are constantly formed in the body and have been implicated in pathogenic states and oxidative stress [4]. Of these ROS, superoxide and hydrogen peroxide radicals are of the most importance in reduction-oxidation reactions as well as substrates in the formation of other ROS, particularly the highly toxic hydroxyl radical [3].

This is due to evidence that $\mathrm{OH} \cdot$ attacks all proteins, DNA, PUFA in cell membranes, and a variety of other molecules [4]. Hydroxyl radicals can be generated by Fenton chemistry

$\left(\mathrm{Fe}^{2+}+\mathrm{H}_{2} \mathrm{O}_{2} \rightarrow \mathrm{Fe}^{3+}+\mathrm{OH}+{ }^{-} \mathrm{OH}\right)$, which are catalyzed by transition metals. Fenton reaction generation of $\mathrm{OH}$. radicals is known to occur in submitochondrial particles under oxidative stress [5] as well as during a number of toxicological states [6]. Exposure to radiation can cause homolytic fission of water molecules, resulting in the production of hydroxyl radicals as well [3].

Reduction-oxidation reactions with superoxide, hydrogen peroxide, and hypochlorous acid also generate hydroxyl radicals in vivo [7].

Superoxide radicals are normally generated in the body via respiratory burst during phagocytosis in immunological defense and from the leakage of electrons from the mitochondrial electron transport chain. Superoxide is an oxygen-centered radical and can have selective reactivity to a variety of tissue types, proteins, DNA, etc. [4]. In addition to superoxide radicals, hydrogen peroxide can be produced during mitochondrial respiration and during the xanthine/ hypoxanthine reaction during purine degradation [8]. $\mathrm{H}_{2} \mathrm{O}_{2}$ can also be formed when superoxide is dismutated (a reaction involving a single substance that produces two products), by the enzyme, superoxide dismutase. $\mathrm{H}_{2} \mathrm{O}_{2}$ is comparatively weakly reactive [4], however it can be a substrate for hydroxyl radical production as well as generation of other ROS.

The ground state of oxygen is essential to all aerobic organisms. However, oxygen reliance can lead to toxicity and imbalance in the body's physiological processes. Oxidative stress is one such instance in which this toxicity occurs. Oxidative stress can be defined as an 
imbalance between the oxidants (ROS) in the body and the antioxidants, such that the imbalance favors the oxidants [9]. The ROS imbalance can be caused, in general, by diminished antioxidant concentration and by increased production of ROS [3]. This can be caused by endogenous sources such as the leak of electrons from mitochondria [10] and exogenous sources such as carbon monoxide [6], radiation, and other environmental factors. Accumulated damage by ROS then contributes to many pathogenic states as well as nonpathogenic states due to the highly unstable nature of the ROS. Strand breakage, base modification, and DNA-protein crosslinks can be linked to ROS mediated damage [3].

Oxidative stress has been implied as one of the major contributing factors to DNA damage. DNA damage and repair occur in vivo at a continuous rate [4] with hydroxyl and superoxide radicals implicated in the mechanism behind DNA damage. Single strand-breaks of DNA can be inhibited by antioxidant defense systems such as uric acid [11].

Oxidative stress has also been liked with various disease states such as ischemia and reperfusion, diabetes, and neurodegenerative disease. Ischemia reperfusion occurs during cardiovascular events or damage to the brain in which the tissue is deprived of oxygen [3]. Associated with these events is an increase in ROS as well as an increase in xanthine oxidase activity which leads to the release of superoxide radicals from the reaction with xanthine [12]. It has also been reported that peroxynitrite has a role in CNS inflammation [13] and in multiple sclerosis [14].

Lastly, oxidative stress is a factor in the aging process. Over time, oxidative damage has an effect on the glycoxidation of proteins, lipids, and DNA. Accumulation of these glycoxidation products can impair tissue function and impact the aging process [15]. In an attempt to cope with oxidative stress, the organism typically up-regulates the antioxidant defense systems.

Organisms balance their redox status by mediation of ROS production by antioxidants. An antioxidant is, broadly, an agent that catalytically removes free radicals and actively scavenges ROS and reactive nitrogen species (RNS) [3]. Antioxidants tend to work at low concentrations and are readily oxidized by ROS thereby decreasing the ability of ROS to react with surrounding cells and tissues. It has also been suggested that individual low molecular weight antioxidants participate in a coordinated network with other antioxidants that scavenge radicals [16].

\section{Antioxidant Defense System: Selected antioxidants and uric acid}

Antioxidants and nutrition form an intimate relationship. Exogenous sources of antioxidants are those that can be obtained from the diet. These can include, but are not limited to, Vitamin $\mathrm{E}$ (tocopherols), ascorbic acid (Vitamin C), carotenoids and flavonoids.

Vitamin E ( $\alpha$-tocopherol) is a potent membrane-bound, lipid soluble antioxidant that is known to react in a direct manner with singlet oxygen [19]. Vitamin $\mathrm{E}$ is also known as a scavenger of peroxyl radicals. It is thought that $a$-tocopherol has the most potent antioxidant capabilities compared to other tocopherols due to the fact that the $\mathrm{H}+$ donating ability of different tocols increases in efficiency with greater ring methyl substitution [17]. During a deficiency state, it has been reported that low vitamin E status resulted in the depletion of other antioxidants such as ascorbate [18]. It was also reported that mice with single-walled carbon nanotubes inserted in the lung showed induced accumulation of lipid peroxidase products and a more severe oxidative stress state due to deficiency in vitamin E [18]. Vitamin E is decidedly an important antioxidant along with vitamin C.

Vitamin C (ascorbic acid or ascorbate) is also an exogenous antioxidant that can be derived from the diet. Vitamin $\mathrm{C}$ is an important water-soluble cytosolic antioxidant involved with radical chain-breaking and regeneration of tocopherol from tocopheroxy radicals [19]. With the information concerning the regeneration of tocopherol from the tocopheroxy radical, it has been reported that there is a synergistic relationship between vitamin $\mathrm{C}$ and vitamin $\mathrm{E}$ [20]. Vitamin C scavenges superoxide radicals as well as other singlet oxygen species [19]. In patients suffering an acute stroke it is often noted that there is a reduction of vitamin $\mathrm{E}$ and ascorbate, which links a decrease in antioxidant defense with a higher incidence of oxidative damage [21].

In addition to dietary antioxidants, endogenous (genetic) antioxidants also play a vital role in regulating ROS production and can be either enzymatic or non-enzymatic. One of the most wellknown of these is the enzyme, superoxide dismutase (SOD). SOD is found in the cytosol (CuZn SOD) of cells as well as in the mitochondrial matrix (Mn-SOD) [22]. As SOD scavenges superoxide, it limits the production of other ROS. In addition to enzymatic endogenous antioxidants, the body also has non-enzymatic antioxidant defense systems.

Uric acid is the end-product of purine metabolism in humans, reptiles, new world primates, and birds. Unlike most mammals, these species lack uricase or urate oxidase (Uox) which catalyzes the oxidation of uric acid to allantoin. Analysis of the promoter, coding, exonic and intronic regions of human and several primate species determined that the hominoid lineage had eight independent nonsense mutations that resulted in the deactivation of the Uox gene [23]. Of the eight mutations, six are caused by a change from $\mathrm{C}$ to $\mathrm{T}$ in the arginine codon (CGA). After examination of the prevalence of the arginine codon in the primate species, it was suggested that the increasing occurrence of the CGA codon is highly correlated with the loss of urate oxidase [23]. The authors concluded that this loss was a stepwise event and not a single step over evolutionary time [23]. Currently, the DNA database does not have a Uox sequence for birds or reptiles and further analysis will be needed to discover the evolutionary loss of Uox in these species. The loss of this enzyme activity by long-lived species is linked to the antioxidant properties of uric acid. It is also hypothesized that coevolution occurred between the down regulation of XOR and the increase in uric acid concentrations in species lacking urate oxidase [23]. The transcription and core promoter activity of human XOR is repressed [24], suggesting that there is a regulatory mechanism to prevent the overproduction of uric acid in purine metabolism. It should be determined if such a relationship exists in species other than humans that lack the same Uox activity and whether this is, in fact, a coevolutionary event that can be linked to antioxidant defense involving uric acid. An extensive review of the loss of uricase and clinical implications in human pathology has been published [25]

Uric acid is a potent plasma antioxidant (Figure 1) that scavenges singlet oxygen, peroxy radicals, and hydroxyl radicals [1] and has been studied extensively in many physiological and pathological systems including neurodegenerative diseases. Human plasma uric acid concentrations are typically high $(\geq 2 \mathrm{mg} / \mathrm{dL})$ with a mean of $7.0 \mathrm{mg} / \mathrm{dL}$ in men and $6.0 \mathrm{mg} / \mathrm{dL}$ in women $[21,26]$. Uric acid can also bind with iron ion complexes, which could signify additional antioxidant 
capabilities [27]. Studies have demonstrated the free radical scavenging abilities of uric acid, in doses equal to and exceeding the range found in birds, using electron spin resonance [28]. Uric acid had a concentration-dependent effect on reducing hydroxyl and superoxide radicals such that increasing the concentration of uric acid increased scavenging of these radicals [28]. Further evidence from this study showed a decrease in DNA fragmentation and lipid peroxidation [28]. These results support the argument that uric acid acts as a scavenger of free radicals and can be considered vital to antioxidant defense.

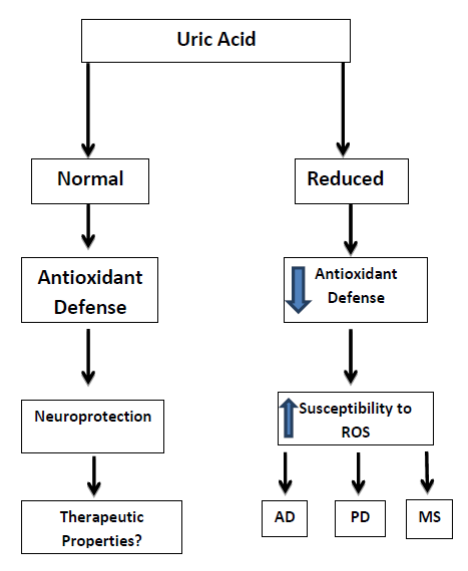

Figure 1: Proposed antioxidant properties of uric acid as it pertains to pathogenesis of some neurodegenerative diseases. Uric acid is considered a potent antioxidant that has been demonstrated to be neuroprotective. In the case of neurodegenerative disease pathogenesis, studies have shown that a reduction of uric acid is linked to an increased propensity toward disease progression. Due to the antioxidant nature of uric acid, it can be proposed that uric acid be considered as a therapeutic agent.

Notably, there are several comparative studies in birds that demonstrate a direct correlation between increased uric acid concentrations and a reduction in oxidative stress and vice versa [29, 30]. Broilers fed a high protein diet consisting of $45 \%$ casein had a significant increase in plasma uric acid concentrations, which was associated with a decrease in oxidative stress in the fast growing birds suggesting that the high protein levels were not detrimental to bird health [31]. When broilers were fed diets containing either xanthine or hypoxanthine, plasma uric acid was markedly increased as compared to controls, which was associated with a decrease in oxidative stress [30]. In contrast, birds fed allopurinol ( $50 \mathrm{mg} / \mathrm{kg}$ body mass) showed decreased plasma uric acid concentrations associated with a significant increase in oxidative stress [30]. Associated with the increase in oxidative stress was an increase in the expression of IL- $1 \beta$, IFN- $\gamma$ and IL-12p35 (Settle, unpublished observations), indicative of a proinflamatory response. A related study with inosine $(0.6 \mathrm{moles} / \mathrm{kg}$ feed/day) administered to broilers treated with hemin (a prooxidant) demonstrated that inosine elevated plasma uric acid concentrations and reduced hemin-induced oxidative stress [32]. When a mixture of inosine and allopurinol were fed to broilers for 3 days there was a marked decrease in plasma uric acid as well as tissue uric acid as compared with broilers receiving only inosine, which suggests that tissues may be susceptible to oxidative damage even after removal of allopurinol from the diet [33]. Conclusions from these studies support the view that the inverse relationship between uric acid concentrations and oxidative stress are valid and comparative across species.

\section{Multiple Sclerosis}

Uric acid also has a role in protecting DNA from single-strand breaks caused by free radicals in the body [11] as well as a protective role in neurodegenerative diseases. Uric acid, as a scavenger of peroxynitrite, has been shown to exhibit protective properties in the inhibition of CNS inflammation and the blood-CNS barrier that has been compromised by peroxynitrite damage [13]. In addition, there was a reduction in encephalomyelitis after treatment with uric acid in the symptoms and increased survivability in a mouse model for multiple sclerosis [14]. Multiple sclerosis (MS) patients are known to have lower serum uric acid concentrations and an increase in oxidative stress via the production of nitric oxide and the up-regulation of iNOS whereas patients with gout have been reported not to have MS [35]. Gout and multiple sclerosis are mutually exclusive, which suggests that reduced uric acid can be linked to a greater susceptibility to neurodegenerative disease progression due to the lack of a potent antioxidant defense mechanism whereas treatment with uric acid precursors such as inosine may have therapeutic benefits. Plasma uric acid concentrations increased from $4 \mathrm{mg} / \mathrm{dl}$ to $9 \mathrm{mg} / \mathrm{dl}$ in patients with diagnosed chronic MS and administered inosine in 1-3 gm increments twice daily, which resulted in a reduction in symptoms with no signs of relapse over a one year period [36]. Furthermore, these patients showed no adverse side effects to the inosine administration, indicating that purine precursors show potential as therapeutic treatments of MS.

\section{Hypoxia and Ischemic Stroke}

Hypoxia and ischemic stroke are both conditions during which tissue is deprived of oxygen for a certain amount of time before restoration. During this time, cell death and apoptosis are occurring with free radical damage linked to many of the complications associated with reperfusion of the tissue. The mechanisms for this have been reviewed extensively [35] although a role of uric acid in ischemic events is not fully understood. Studies in hippocampal cell culture found that uric acid protects against metabolic insults and attenuates oxidative damage [37]. It has also been reported that rats exposed to ischemia/reperfusion during and following treatment with uric acid have shown reduced markers of injury to the brain as well as amelioration of behavioral and cognitive deficits often associated with these injuries [37]. Furthermore, the combination of uric acid with a recombinant tissue plasminogen activator (rt-PA) in ischemic stroke patients, prevented the early fall of UA associated with a loss of neural protection [38]. In addition to ischemic injury, there are studies supporting the neuroprotective properties of uric acid in neurodegenerative diseases such as Parkinson's and Alzheimer's.

\section{Parkinson's Disease}

Parkinson's Disease (PD) is a neurodegenerative disease that may in fact be correlated with uric acid concentrations. PD is characterized by degeneration of the pigmented dopaminergic neurons in the substantia nigra pars of the mid brain. Patients will present with bradykinesia, resting tremors, and cognitive impairment among other symptoms. Although the specific underlying cause of this cell death is not fully understood it is hypothesized that oxidative stress and 
mitochondrial dysfunction may play a role in the pathogenesis of PD and this has been reviewed in detail [39]. The role of uric acid with the pathogenesis of PD has not been fully elucidated, but evidence suggests that decreased serum uric acid concentrations have been linked with disease progression.

In a population study, 4,695 participants screened for PD and patients with Parkinson's or dementia markers at baseline for each disease were excluded from the data [40]. Results from this study showed that there was a correlation between higher serum uric acid concentrations and a reduced incidence of PD over a course of 9.4 years [40]. Additionally, case studies similarly showed that higher serum levels of uric acid are associated with a decrease in the development of PD [41, 42]. Cognitive impairment is also associated with PD. There was a correlation between cognitive ability with $40 \mathrm{PD}$ patients such that diminished cognitive exams were associated with reduced plasma uric acid levels [43]. Based on these findings, it has been suggested that uric acid be considered for therapeutic use in patients with $\mathrm{PD}$ however, more research is needed in this area to ascertain the dose of UA and how this will ultimately affect overall physiological response.

\section{Alzheimer's Disease}

Alzheimer's disease (AD) is also characterized by a loss of cognitive function and neuronal degeneration in patients. $\mathrm{AD}$ is ultimately considered a neuropsychiatric disorder and the etiology of the disease remains largely unknown. Oxidative stress induced damage by enhanced lipid peroxidation in areas of the brain have been implicated as an underlying factor in disease progression [44]. Free radical damage may also be a factor in $\beta$-amyloid plaque formation associated with AD [45]. There is an increase of antioxidants such as catalase and SOD in the hippocampus and amygdala during AD [46, 47]. Similar to $\mathrm{PD}$, uric acid is decreased in patients with $\mathrm{AD}[48,49]$. Research has suggested that combining uric acid precursors such as inosine with ascorbic acid may have therapeutic benefits for AD patients, but dose and duration of treatment have not been determined. $\mathrm{AD}$ is a progressive debilitating disease that may, in the future, also incorporate inosine as a therapeutic agent.

\section{Conclusions}

Oxidative damage plays a role in the progression of neurodegenerative disease states and injury to neural tissue. The brain and central nervous system are exposed to oxidative damage generated by free radical processes throughout life. It is hypothesized that this constant assault by ROS on these tissues can greatly contribute to the pathogenesis of certain disease states and exacerbate injury sites. Understanding the antioxidant defense system is critical in finding markers of disease as well as potential therapeutic treatments. Uric acid is a potent endogenous antioxidant for humans, birds, and reptiles. Comparative studies in birds demonstrate that a reduction of uric acid leads to an increase in inflammation and oxidative stress that can be ameliorated by administration of purine precursors. Similarly, this treatment is also suggested for patients suffering from MS, AD, $\mathrm{PD}$, and ischemic events. Furthermore, evidence presented in the literature demonstrates, consistently, that uric acid is lower in patients with these neurodegenerative events (figure 1), which suggests that the neurological system relies on uric acid to mediate free radical damage through the scavenging of peroxynitrite. While there is a plethora of information about uric acid, there is much to learn about its role in the antioxidant defense system and the potential use of uric acid or its precursors in the prevention and treatment of disease.

\section{Acknowledgements}

We would like to thank Dr. Ken Blemings for his review of the manuscript.

\section{References}

1. Ames BN, Cathcart R, Schwiers E, Hochstein P (1981) Uric acid provides an antioxidant defense in humans against oxidant- and radical-caused aging and cancer: a hypothesis. Proc Natl Acad Sci U S A 78: 6858-6862.

2. Fang YZ, Yang S, Wu G (2002) Free radicals, antioxidants, and nutrition. Nutrition 18: 872-879.

3. Halliwell B, Gutteridge JMC (1999) Free Radicals in Biology and Medicine third edition. Oxford University Press.

4. Aruoma O (1998) Free radicals, oxidative stress, and antioxidants in human health and disease. JAOCS $75: 199-211$

5. Thomas C, Mackey MM, Diaz AA, Cox DP (2009) Hydroxyl radical is produced via the Fenton reaction in submitochondrial particles under oxidative stress: implications for diseases associated with iron accumulation. Redox Report 14: 102-108.

6. Hara S, Mizukami H, Mukai T, Kurosaki K, Kuriiwa F, et al. (2009) Involvement of extracellular ascorbate and iron in hydroxyl radical generation in rat striatum in carbon monoxide poisoning. Toxicology 264: 69-73.

7. Rosen GM, Pou S, Ramos CL, Cohen MS, Britigan BE (1995) Free radicals and phagocytic cells. FASEB J 9: 200-209.

8. Terada LS, Leff JA, Repine JE (1990) Measurement of xanthine oxidase in biological tissues. Methods Enzymol 186: 651-656.

9. VanDyke K, VanDyke C, Woodfork K (2002) Luminescence Biotechnology Instruments and Applications. CRC Press LLC.

10. Cadenas E, Davies KJ (2000) Mitochondrial free radical generation, oxidative stress, and aging. Free Radic Biol Med 29: 222-230.

11. Cohen AM, Aberdroth RE, Hochstein P (1984) Inhibition of free radicalinduced DNA damage by uric acid. FEBS Lett 174: 147-150.

12. Harrison R (2002) Structure and function of xanthine oxidoreductase: where are we now? Free Radic Biol Med 33: 774-797.

13. Hooper DC, Scott GS, Zborek, A, Miksheeva T, Kean RB (2000) Uric acid, a peroxynitrite scavenger, inhibits CNS inflammation, blood-CNS barrier permeability changes, and tissue damage in a mouse model of multiple sclerosis. FASEB J. 14: 691-698

14. Hooper DC, Spitsin S, Kean RB, Champion JM, Dickson GM, et al. (1998) Uric acid, a natural scavenger of peroxynitrite, in experimental allergic encephalomyelitis and multiple sclerosis. Proc Natl Acad Sci U S A 95: 675-680.

15. Martin GM, Austad SN, Johnson TE (1996) Genetic analysis of ageing: role of oxidative damage and environmental stresses. Nat Genet 13: 25-34.

16. Kagan VE, Packer L (1994) Light-induced generation of vitamin E radicals: assessing vitamin $\mathrm{E}$ regeneration. Methods Enzymol 234: 316-320.

17. Traber MG, Atkinson J (2007) Vitamin E, antioxidant and nothing more. Free Radic Biol Med 43: 4-15.

18. Shvedova A, Kisin E, Murray A, Gorelik O, Arepalli S et al. (2007) Vitamin E deficiency enhances pulmonary inflammatory response and oxidative stress induced by single-walled carbon nanotubes in C57BL/ 6 mice. Toxicology and Applied Pharmacology 221: 339-348

19. Machlin LJ, Bendich A (1987) Free radical tissue damage: protective role of antioxidant nutrients. FASEB J 1: 441-445.

20. Niki E (1991) Action of ascorbic acid as a scavenger of active and stable oxygen radicals. Am J Clin Nutr 54: 1119S-1124S. 
21. Chamorro A, Planas AM, Muner DS, Deulofeu R (2004) Uric acid administration for neuroprotection in patients with acute brain ischemia. Med Hypotheses 62: 173-176.

22. Turrens JF (2003) Mitochondrial formation of reactive oxygen species. J Physiol 552: 335-344.

23. Oda M, Satta Y, Takenaka O, Takahata N (2002) Loss of urate oxidase activity in hominoids and its evolutionary implications. Mol Biol Evol 19: 640-653.

24. Xu P, LaVallee P, Hoidal JR (2000) Repressed expression of the human xanthine oxidoreductase gene. E-box and TATA-like elements restrict ground state transcriptional activity. J Biol Chem 275: 5918-5926.

25. Álvarez-Lario B, Macarrón-Vicente J (2010) Uric acid and evolution. Rheumatology (Oxford) 49: 2010-2015.

26. Fang P, Li X, Luo JJ, Wang H, Yang XF (2013) A Double-edged Sword: Uric Acid and Neurological Disorders. Brain Disord Ther 2: 109.

27. Davies KJ, Sevanian A, Muakkassah-Kelly SF, Hochstein P (1986) Uric acid-iron ion complexes. A new aspect of the antioxidant functions of uric acid. Biochem J 235: 747-754.

28. Stinefelt B, Leonard SS, Blemings KP, Shi X, Klandorf H (2005) Free radical scavenging, DNA protection, and inhibition of lipid peroxidation mediated by uric acid. Ann Clin Lab Sci 35: 37-45.

29. Carro MD, Falkenstein E, Radke W.J, Klandorf H (2009) Effects of allopurinol on uric acid concentrations, xanthine oxidoreductase activity, and oxidative stress in broiler chickens. Comparitive Biochemistry and Physiology, Part C 151: 12-17

30. Simoyi MF, Van Dyke K, Klandorf H (2002) Manipulation of plasma uri acid in broiler chicks and its effect on leukocyte oxidative activity. Am J Physiol Regul Integr Comp Physiol 282: R791-796.

31. Machin M, Simoyi M, Blemings K, Klandorf H (2004) Increased dietary protein elevates plasma uric acid and is associated with decreased oxidative stress in rapidly-growing broilers. Comparative Biochemistry and Physiology Part B. 137: 383-390.

32. Seaman C, Moritz J, Falkenstein E, Van Dyke K, Casotti G, et al. (2008) Inosine ameliorates the effects of hemin-induced oxidative stress in broilers. Comp Biochem Physiol A Mol Integr Physiol 151: 670-675.

33. Settle T, Carro MD, Falkenstein E, Radke W, Klandorf H (2012) The effects of allopurinol, uric acid, and inosine administration on xanthine oxidoreductase activity and uric acid concentrations in broilers. Poult Sci 91: 2895-2903.

34. Sotgiu S, Pugliatti M, Sanna A, Sotgiu A, Fois ML, et al. (2002) Serum uric acid and multiple sclerosis. Neurol Sci 23: 183-188.

35. EreciÅ ska M, Silver IA (2001) Tissue oxygen tension and brain sensitivity to hypoxia. Respir Physiol 128: 263-276.

36. Yu Z.F, Annadora J, Bruce-Keller Y, Goodman Y, Matteson MP (1998) Uric acid protects neurons against excitotoxic and metabolic insults in cell cultura, and against focal ischemic brain injury in vivo. Journal of Neuroscience Research 53: 613-625

37. Amaro S, Soy D, Obach V, Cervera A, Planas AM, et al. (2007) A pilot study of dual treatment with recombinant tissue plasminogen activator and uric acid in acute ischemic stroke. Stroke 38: 2173-2175.

38. Hald A, Lotharius J (2005) Oxidative stress and inflammation in Parkinson's disease: is there a causal link? Exp Neurol 193: 279-290.

39. de Lau LM, Koudstaal PJ, Hofman A, Breteler MM (2005) Serum uric acid levels and the risk of Parkinson disease. Ann Neurol 58: 797-800.

40. Davis JW, Grandinetti A, Waslien CI, Ross GW, White LR, et al. (1996) Observations on serum uric acid levels and the risk of idiopathic Parkinson's disease. Am J Epidemiol 144: 480-484.

41. Weisskopf MG, O'Reilly E, Chen H, Schwarzschild MA, Ascherio A (2007) Plasma urate and risk of Parkinson's disease. Am J Epidemiol 166 561-567.

42. Annanmaki T, Muuronen A, Murros K (2007) Low plasma uric acid level in Parkinson's disease. Mov Disord 22: 1133-1137.

43. Lovell MA, Ehmann WD, Butler SM, Markesbery WR (1995) Elevated thiobarbituric acid-reactive substances and antioxidant enzyme activity in the brain in Alzheimer's disease. Neurology 45: 1594-1601.

44. Pappolla MA, Chyan YJ, Omar RA, Hsiao K, Perry G, et al. (1998) Evidence of oxidative stress and in vivo neurotoxicity of beta-amyloid in a transgenic mouse model of Alzheimer's disease: a chronic oxidative paradigm for testing antioxidant therapies in vivo. Am J Pathol 152: 871-877.

45. Zemlan FP, Thienhaus OJ, Bosmann HB (1989) Superoxide dismutase activity in Alzheimer's disease: possible mechanism for paired helical filament formation. Brain Res 476: 160-162.

46. Pappolla MA, Omar RA, Kim KS, Robakis NK (1992) Immunohistochemical evidence of oxidative [corrected] stress in Alzheimer's disease. Am J Pathol 140: 621-628.

47. Hensley K, Maidt M, Yu Z, Sang H, Markesbery W et al. (1998) Electrochemical analusis of protein nitrotyrosine and dityrosine in Alzheimer brain indicates region-specific accumulation. Journal of Neuroscience 18: 8126-8132

48. Kim TS, Pae CU, Yoon SJ, Jang WY, Lee NJ, et al. (2006) Decreased plasma antioxidants in patients with Alzheimer's disease. Int J Geriatr Psychiatry 21: 344-348.

49. Waugh WH (2008) Inhibition of iron-catalyzed oxidations by attainable uric acid and ascorbic acid levels: therapeutic implications for Alzheimer's disease and late cognitive impairment. Gerontology 54: 238-243. 